\title{
On-Line Analysis of the Heterogeneous Pd-Catalyzed Transfer Hydrogenation of $p$-Nitrophenol in Water with Formic Acid in a Flow Reactor
}

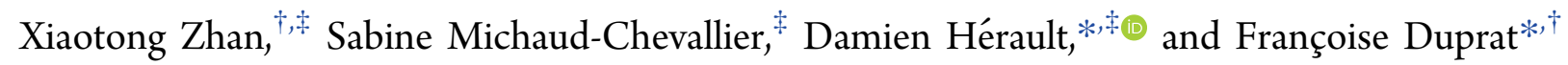

\begin{abstract}
An versatile experimental setup composed of a fixed-bed reactor and an HPLC apparatus has been developed for the study of heterogeneous catalytic reactions under transient conditions. The transfer hydrogen reaction of formic acid in water to give $p$-nitrophenol catalyzed by $\mathrm{Pd} @ \mathrm{Al}_{2} \mathrm{O}_{3}$ support was chosen as the model reaction in a flow reactor. On one hand, this setup allowed the first experimental on-line measurements of $p$-aminophenol in an acidic medium, indicating that it is the only product of the reaction at steady state. On the other hand, the setup evidenced a quite long transient period before stationary conditions were reached, involving a transient component that is quite instable and can transform to $p$-aminophenol without a catalyst. Results of numerical simulations indicate that only a change in the reaction mechanism, due to a change in the catalyst activity after few minutes of reaction, can explain the transient production of this intermediate and that this occurs when the active hydrogen coverage of the catalyst is low.
\end{abstract}

\section{INTRODUCTION}

The use of a flow reactor as a lab tool either for optimization of reaction conditions or for determination of the rate equation implies frequent changes in operating conditions. ${ }^{1}$ Therefore, the period between experiment startup and achievement of the steady state is important to control in order to minimize the optimization period. In the case of heterogeneous catalysis, adsorption on the catalyst support can lead to an accumulation of reactant within the reactor. ${ }^{2}$ Thus, the time to achieve the steady state may be much longer than the liquid residence time (calculated as the fluid volume divided by the flow rate). Online analysis of the reactor effluent may be useful to assess the impact of a change in operating conditions on the extent of the reaction. In addition, in the case of complex reactions, individual quantification of reactants and products flowing out of the reactor may be necessary. Here we present a polyvalent combination of a flow reactor and a UHPLC device that allows either on-line UV-vis continuous analysis or online chromatographic analysis of the reaction mixture at both the entrance and outlet of the reactor. The transfer hydrogenation of $p$-nitrophenol (1) with formic acid (FA) as the hydrogen source is a perfect reaction to exemplify interest in on-line analysis from both the academic and industrial points of view. ${ }^{3}$ Actually, although it is considered to be simple because aminophenol is usually the only product, the reaction may proceed via two reaction routes, the direct one and the condensation one, and involves several intermediate species, among them hydroxylamine 4, which can accumulate on the oxide catalyst support (Figure 1 ). ${ }^{4}$

In addition, transfer hydrogenation of nitroarenes with $\mathrm{NaBH}_{4}$ as the hydrogen source has been widely utilized as a model reaction to check the catalytic activity of heterogeneous catalysts because of analysis facility. ${ }^{5}$ Actually, under the large stochiometric excess of $\mathrm{NaBH}_{4}$ usually employed, the extent of reaction can be easily monitored by UV-vis spectroscopy because of the strong absorption of 4-nitrophenolate ions at $400 \mathrm{~nm}$ and the distinct band of 4-aminophenol (2) at 300 $\mathrm{nm} .^{5 \mathrm{~b}-\mathrm{k}, \mathrm{m}}$ However, under the acidic conditions used by Javaid and Suzuki, the maximum absorbances of $\mathbf{1}$ and $\mathbf{2}$ both shift slightly with variation of the $\mathrm{pH} .{ }^{6}$ Therefore, UV monitoring of the reaction mixture gives only a not very accurate evaluation of the consumption of $\mathbf{1}$.

Aniline derivatives are important building blocks in the synthesis of pharmaceuticals, agricultural chemicals, and other fine chemicals. The reduction of $\mathbf{1}$ to $\mathbf{2}$ is the main route for the production of paracetamol ( $N$-acetyl-4-aminophenol) in Asia. ${ }^{8}$ The reaction is considered to be a useful transformation both in the pharmaceutical industry and in the pollutantremediation field, as $\mathbf{1}$ is a toxic byproduct of pesticide and dye production. Industrial production involves direct catalytic hydrogenation with $\mathrm{H}_{2}$ at relatively high pressure and temperature. Recently it has been demonstrated that FA can be used as a hydrogen source for the transfer hydrogenation of nitroarenes, ${ }^{9}$ notably for the reduction of $\mathbf{1}$ in aqueous media on palladium-coated tubes. ${ }^{6}$ A fundamental study of hydrogen generation from formic acid decomposition on the (211) faces of $\mathrm{Ni}, \mathrm{Pd}$, and Pt showed that FA adsorption is an exothermic and thermodynamically favorable process. Formate dissociation into $\mathrm{CO}_{2}$ and surface $\mathrm{H}$, which is the rate-determining 


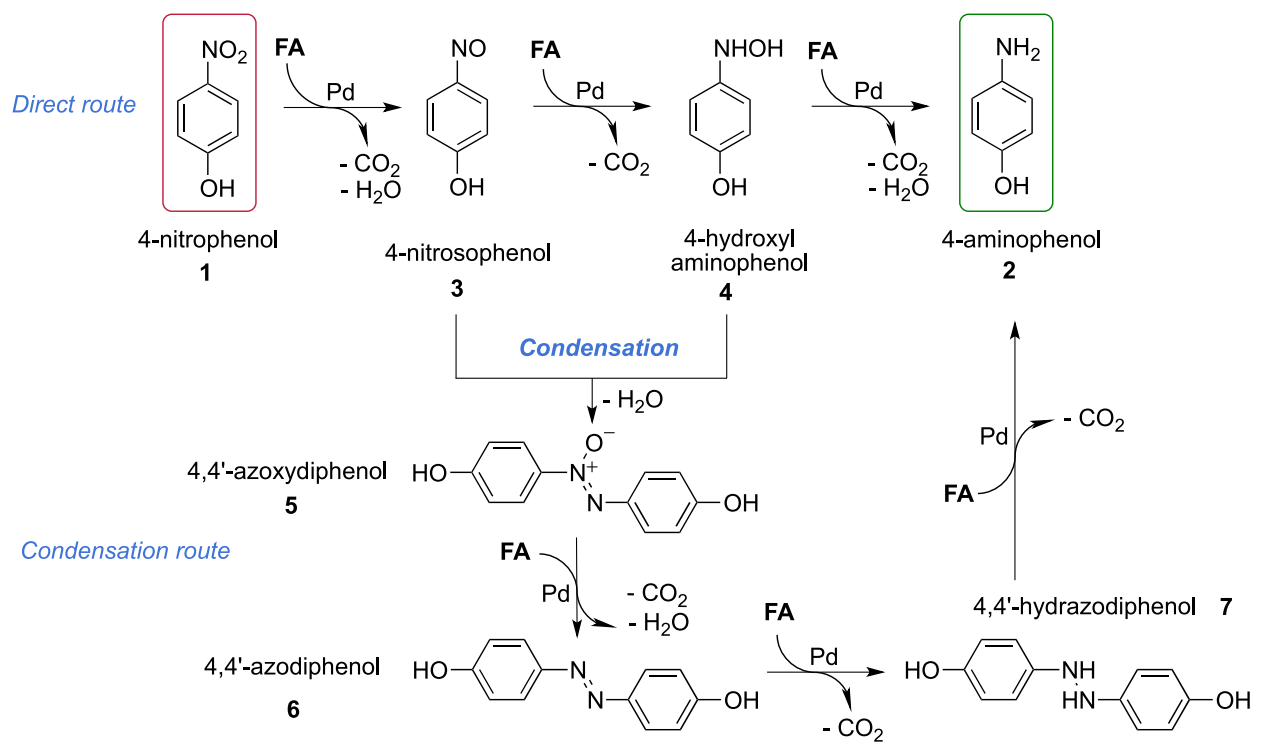

Figure 1. Proposed mechanism for the hydrogenation of $p$-nitrophenol with formic acid on a Pd catalyst (adapted from Blaser ${ }^{4}$ for $p$-nitrophenol).

step, depends on the face concerned and is exothermic on $\operatorname{Pd}(211)$ and $\operatorname{Pt}(211) .{ }^{10}$ FA is inexpensive, easily handled, and abundantly available. Thus, from an industrial point of view, catalytic transfer hydrogenation of nitroarenes with FA provides an efficient and environmentally benign alternative to the reduction with $\mathrm{H}_{2}$. Beller and co-workers indicated that FA deactivates non-noble metal catalysts through corrosion or leaching problems. ${ }^{3}$ Therefore, except for a study based on $\mathrm{Au},{ }^{9 \mathrm{~d}}$ most of catalysts involved in transfer hydrogenation of nitroarenes with FA are Pd-based. Their activity is excellent under soft conditions (aqueous phase, $30-50{ }^{\circ} \mathrm{C}$ ). ${ }^{6,11}$ Actually, a turnover frequency (TOF) of around $320 \mathrm{~h}^{-1}$ was obtained in a tubular reactor wall-coated with porous $\mathrm{PdO}^{6}$ and a TOF of about $1000 \mathrm{~h}^{-1}$ on a photoinduced $\mathrm{Pd} / \mathrm{CN}$ catalyst. ${ }^{9 \mathrm{e}}$

The present study deals with the transfer hydrogenation of $p$-nitrophenol with aqueous FA catalyzed by $\mathrm{Pd} @ \mathrm{Al}_{2} \mathrm{O}_{3}$ in a flow reactor. A versatile experimental setup composed of a catalytic flow reactor and standard HPLC equipment was developed for this purpose. As we observed an unexpected transient component in the reaction mixture, an extensive bibliographic study of the mechanism of transfer hydrogenation of nitroarene is first summarized. Then spectroscopic and chromatographic experimental results related to the transient period until achievement of the steady state are presented and compared with patterns of the time evolution of concentrations obtained by numerical simulation. The last part is devoted to attempts to identify the transient component.

\section{TRANSFER HYDROGENATION OF NITROARENES}

In a large review of selective catalytic hydrogenation of nitroarenes, Zou and co-workers analyzed the mechanisms of both activation of the nitro group and production of active hydrogen species. ${ }^{12}$ The reaction mechanism for the catalytic hydrogenation of nitrobenzene, proposed by Haber et al. more than 100 years ago, is generally accepted to describe how the transfer hydrogenation proceeds. Haber's mechanism involves a direct route and a condensation route (Figure 1, adapted for 1).

Byproducts or intermediates are rarely detected in the reaction mixture, and the amine is practically the only observable product of the reaction. Several kinetic studies involving different metal nanoparticles as catalysts supposed that both reactants (the hydrogen donor and the nitroarene) must be adsorbed on the catalyst to react. ${ }^{5 e, f}$ Experimental evidence was provided by Corma and co-workers, who realized the reaction in a FTIR cell and showed that the nitroso and hydroxylamine react before desorption and that the last step leading to the amine is the rate-determining step. ${ }^{13}$ This was also evidenced by the fact that the reaction rates of nitrobenzene and hydroxylaminobenzene with aniline are comparable. ${ }^{14}$ In some cases, with Pt-based catalysts, accumulation of hydroxylamine was reported. ${ }^{3}$ Hydrogenation reactions of intermediates of the reaction give a more complex picture than Haber' mechanism. Jackson and co-workers compared the isotope effect of $\mathrm{H}_{2}$ and $\mathrm{D}_{2}$ and observed opposite effects on nitro- and nitrosobenzene. ${ }^{15}$ In addition, the reaction rate of nitrosobenzene was 5 times lower than that of nitrobenzene and led to an azobenzene/aniline mixture, contrarily to nitrobenzene. The authors also proposed the formation of 4-hydroxylaminobenzene radical (8) as the key intermediate (Figure 2).

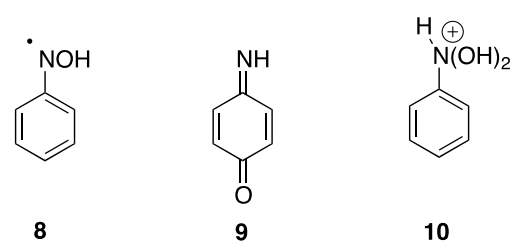

Figure 2. Plausible intermediates described in the literature.

Corma and co-workers ${ }^{13}$ observed a similar trend: the formation of hydroxylamine is faster starting from nitrobenzene than from nitrosobenzene. They also concluded that there are two parallel routes in the direct pathway of hydrogenation: formation of phenylhydroxylamine is both a primary product (from nitrobenzene) and a secondary product (via nitrosobenzene). Theoretical considerations gave interesting insight into the reaction mechanism and could explain the previous observations. According to density functional theory calculations by Cao and co-workers, the reaction route could be influenced by the nitro group adsorption configuration: 

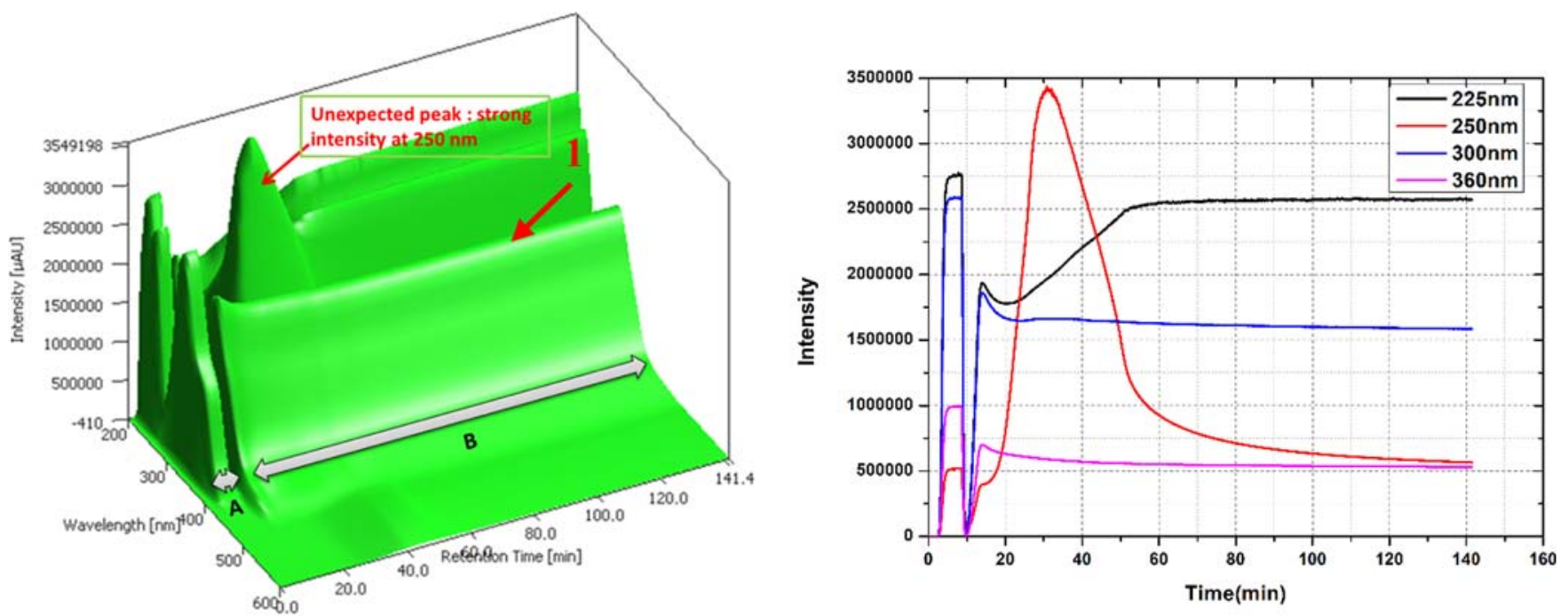

Figure 3. Example UV analysis during a reaction test. (left) 3D (time-wavelength-intensity) representation: (A) reactor feed; (B) reactor effluent. (right) $2 \mathrm{D}$ (time-intensity) plot. Conditions: $C_{1}^{0}=0.4 \mathrm{mmol} / \mathrm{L} ; C_{\mathrm{FA}}^{0}=1.2 \mathrm{mmol} / \mathrm{L} ; T=40^{\circ} \mathrm{C} ; Q=0.5 \mathrm{~mL} / \mathrm{min}$.
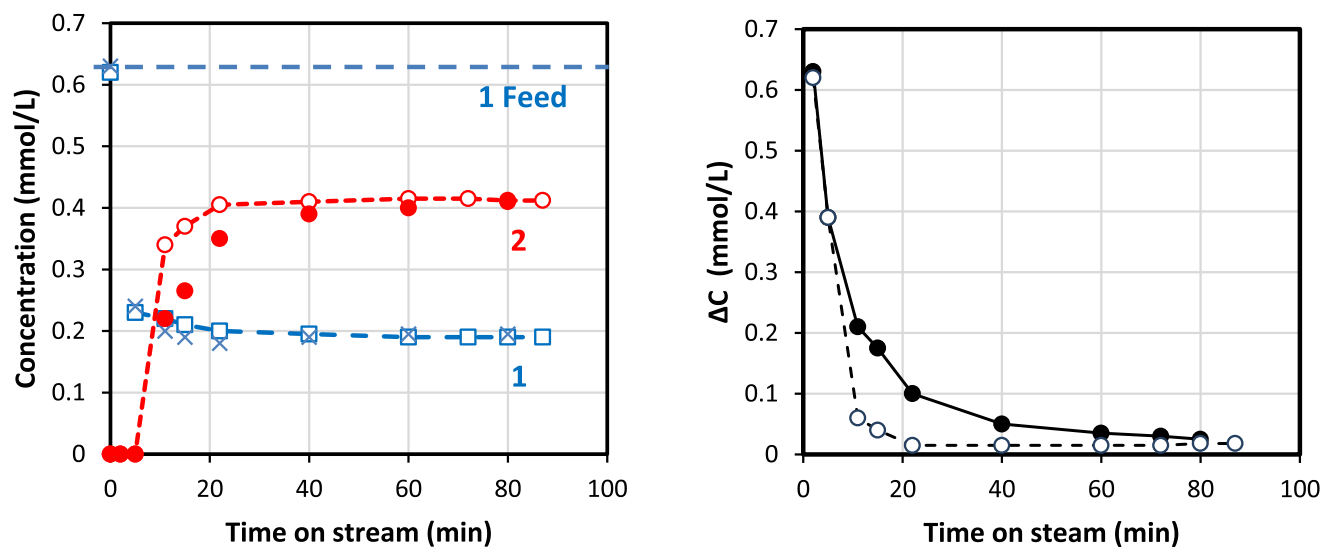

Figure 4. (left) Concentration profiles measured by HPLC analysis: $\square, 1$ on-line; $\times, 1$ off-line; $\mathbf{Q}, 2$ on-line; $\bigcirc, 2$ off-line. (right) Corresponding deviations of the mass balance: $\bullet$, on-line analysis; $\bigcirc$, off-line analysis. Conditions: $C_{1}^{0}=0.6 \mathrm{mmol} / \mathrm{L} ; C_{\mathrm{FA}}^{0}=1.8 \mathrm{mmol} / \mathrm{L} ; T=40{ }^{\circ} \mathrm{C} ; Q=1 \mathrm{~mL} /$ min; steady-state 1 conversion rate $=0.69$.

weak adsorption of $\mathrm{NO}_{2}$ is stabilized by interaction of the aromatic ring with the catalyst, resulting in flat adsorption that makes hydrogenation easier, leading to production of the amine via the direct pathway. ${ }^{16}$ By contrast, strong binding of the NO group leads to vertical adsorption, which is more favorable toward the formation of the azoxy compound. Two other species have been proposed as intermediates in the direct route. Ebitani and co-workers suggested that the transformation of the nitro group to the hydroxylamine could go through $-\mathrm{N}^{+}(\mathrm{OH})_{2} \mathrm{H}$ species 10. ${ }^{9 \mathrm{c}}$ Moreover, several authors cited $p$-benzoquinone imine (9) as an intermediate between the hydroxylamine and amino derivatives (Figure 2). ${ }^{5 i, 17,18} \mathbf{9}$ has been clearly identified as a product of electrochemical reduction of $1,{ }^{19}$ but it is also involved in the oxidation of 2 according to Conant and Pratt. ${ }^{20}$ In acidic media, 9 irreversibly forms a highly colored product in the presence of $\mathbf{2}$. More recently, Lerner identified the structure of this purple dye formed by oxidation of $\mathbf{2}$ in acidic media: two different adducts reflect the UV absorption bands of the dye at 380 and 550 $\mathrm{nm} .^{21}$ Moreover, the formation of the $380 \mathrm{~nm}$ chromogen should be accompanied by the formation of an equivalent amount of 2 . The components involved in the condensation route, such as azo or azoxy, have been evidenced in several studies, and the selectivity toward one or the other route can even be modulated. ${ }^{3,5 \mathrm{~h}, 9 \mathrm{~d}, 12,22}$ Figueras and Coq considered that coupling of the nitroso and hydroxylamine occurs as a fast homogeneous side reaction. At least, condensation means bimolecular nitroso/hydroxylamine interactions. ${ }^{14}$ This contact could be favored either by high concentrations or by weak adsorption allowing mobility of the adsorbed species. Actually, Uberman and co-workers observed that the ratio amine/azoxy is reversed by a change in the amount of catalyst or hydrogen donor, the condensation route being favored by a deficit of these species. ${ }^{51}$ According to Corma, who evidenced the formation of adsorbed intermediates by IR spectroscopy, the support may influence the reaction pathway: the direct route was clearly observed on $\mathrm{Au} @ \mathrm{TiO}_{2}$ and the condensation route on a ceria support because of the higher concentration of adsorbed nitroso. ${ }^{23}$ By contrast, changes in the reaction pathways depending on different amounts of copper in the gold nanoparticles were ascribed by $\mathrm{Zhu}^{5 \mathrm{~h}}$ to weaker adsorption of the nitroarene on $\mathrm{Au}$, favoring the mobility. To the best of our knowledge, only the direct route has been considered in investigations of the transfer hydrogenation with FA on Pd catalysts, which have an excellent activity at temperatures close to ambient. It should be mentioned that at 
higher temperatures $\left(80-120{ }^{\circ} \mathrm{C}\right)$, FA acts as both a hydrogen donor and formylating agent and allows direct synthesis of $\mathrm{N}$ arylformamides, which are valuable intermediates in the synthesis of pharmaceuticals, agrochemicals, dyes, and fragrances. ${ }^{24}$ In the presence of a heterogeneous noble metal catalyst, the reactions are quite slow $(12-24 \mathrm{~h})$, but with aqueous $\mathrm{Pd}(\mathrm{TFA})_{2}$, Lei and co-workers reported yields of 92$99 \%$ within less than 30 min. $^{24 d}$

\section{DYNAMIC STUDY OF CATALYTIC p-NITROPHENOL HYDROGENATION}

Figure 3 exhibits a typical spectrum obtained during the experimental protocol with on-line UV analysis (see Figure S1 for the experimental setup). The 3D (time-wavelengthintensity) representation is presented on the left, and on the right the UV analysis at different wavelengths is shown. It is worth noting that the peak with a strong intensity at $250 \mathrm{~nm}$ is unexpected and is due to the transient presence of an unknown product called $\mathbf{X}$. Further experiments showed that this peak is not an artifact and that it corresponds to a real component that is neither 1 nor 2 . The ridge behind the figure, at $360 \mathrm{~nm}$, corresponds to the profile of $\mathbf{1}$.

The left panel of Figure 4 shows the concentration profiles of 1 and 2 measured by on-line HLPC and confirms the UV profile of $\mathbf{1}$ in Figure 3. 1 flows out of the reactor very quickly, indicating quite poor adsorption of $\mathbf{1}$ on the catalyst when FA is present. The concentration of $\mathbf{1}$ exhibits a shoulder and then stabilizes; meanwhile the concentration of $\mathbf{2}$ slowly increases and reaches the stationary value after a longer time than for $\mathbf{1}$. The left panel of Figure 4 also compares the measurements of the concentration of $\mathbf{2}$, one directly at the exit of the reactor (on-line HPLC; see p S3, protocol B in the Supporting Information) and the other after a delay due to off-line HPLC ( $\mathrm{p} \mathrm{S3}$, protocol A in the Supporting Information): in the first part of the reaction sequence, i.e., between 10-30 min, approximately when $\mathbf{X}$ flows out the reactor, the off-line concentration of $\mathbf{2}$ is significantly higher than the on-line one, while this difference is very small for $\mathbf{1}$ concentrations. This suggests a transformation of $\mathbf{X}$ into $\mathbf{2}$ during the off-line analysis preparation.

The deviation between the quantity of input reactant and output products is calculated as $\left(C_{1}+C_{2}-C_{1}^{0}\right) / C_{1}^{0}$, where $C_{1}^{0}$ is the concentration in the reactor feed and $C_{1}$ and $C_{2}$ are the concentrations of $\mathbf{1}$ and $\mathbf{2}$ at the exit at the time on run under consideration. It can be seen in the right panel of Figure 4 that the deviation of the material balance is low when the steady state is reached. This average deviation over a set of 56 experiments is $-4.0 \%$, which can be considered to be in the experimental error range. Thus, within the measurement precision, the concentration of $\mathbf{2}$ produced is equal to that of 1 reacted, i.e., $\mathbf{2}$ is the only product of the hydrogenation of $\mathbf{1}$. On the contrary, during the period corresponding to the exit of $\mathbf{X}$, the difference between the input and output is up to $33 \%$, and this difference is lower (10\%) when there is a delay between output and analysis. This means that the reaction mixture contains at least one intermediate species able to be transformed in $\mathbf{2}$ without catalyst. Moreover, the observation of $\mathbf{X}$ flowing out of the reactor for a while, clearly indicates a change of the reaction pathway. Indeed, at the beginning of the reaction sequence, $\mathbf{X}$ is formed on the catalyst in sufficient amount to appear out of the reactor, and, after a certain time on stream, $\mathbf{X}$ is no more formed. Different experiments were performed with UV photodiode array (PDA) on-line analysis in order to relate the peak observed at $250 \mathrm{~nm}$ to the experimental conditions (see p 2 in the SI). Its shape and intensity depend on the history of the catalyst. In a series of tests with different washing temperatures and the same reaction conditions, it was observed that the peak intensity depends on the time elapsed between the washing sequence and the reaction sequence (see Figures S2-S4). As a first conclusion, the production of $\mathbf{X}$ is clearly related to the state of the surface of the catalyst, which is the only parameter that was not changed in the experiments. The $\mathbf{X}$ species is no longer formed as soon as enough FA has been introduced, and it is desorbed in order to promote a faster reaction route on the catalytic site.

\section{NUMERICAL SIMULATION OF THE REACTOR}

Numerical simulation of the reactor was performed in order to understand what kind of reaction pattern can produce a transient component in a continuous flow reactor. As only partial quantitative information about the composition of the reaction mixture flowing out of the reactor is available, the simplest model is investigated: an ideal plug-flow model, which is well-suited to fixed-bed catalytic reactors and first-order kinetics. In an ideal plug-flow reactor, the composition of the fluid varies from point to point along the flow path. As the catalyst bed is washed with water between each test, the start of the reaction sequence results in step perturbation at the entrance of the reactor: at first, the catalyst is under pure water, and then at time 0 the reactant mixture is fed at a constant flow rate and composition and reacts in the reactor according to the time evolved since its entrance. As a result, at the early stage, both the composition of the fluid and composition on the catalyst surface vary all along the catalyst bed and with time. Hence, the non-steady-state mass balance over a differential volume of catalyst $\mathrm{d} V_{\text {CAT }}$ during an elementary time interval $\mathrm{d} t$ must be considered for every component $J$ :

$$
-Q \cdot \frac{\partial C_{\mathrm{J}}}{\partial V_{\mathrm{CAT}}}=r_{\mathrm{J}}+\frac{\varepsilon}{1-\varepsilon} \frac{\partial C_{\mathrm{J}}}{\partial t}+\frac{\partial q_{\mathrm{J}}}{\partial t}
$$

where $C_{\mathrm{J}}$ is the molar concentration of component $\mathrm{J}$ in the liquid phase within the reactor, $q_{\mathrm{J}}$ is the molar concentration of $\mathrm{J}$ adsorbed on the catalyst surface, $\mathrm{Q}$ is the volumetric flow rate, $r_{\mathrm{J}}$ is the rate of reaction of component $\mathrm{J}$ per unit catalyst volume, and $\varepsilon$ is the void fraction in the catalyst bed. The boundary conditions are $C_{\mathrm{J}}=q_{\mathrm{J}}=0$ within the reactor at $t=0$ and $C_{1}=C_{1}^{0}$ at the reactor entrance for $t>0$.

Two models of the reaction scheme were studied: one involving an intermediate species in a series of reactions and the other involving a change in the route of reaction due to a change in the surface (see section 3 in the Supporting Information). It appeared that the reaction-in-series model is not able to mimic the observations. The model with a change in the catalyst surface properties is more satisfactory because it roughly represents the pattern of experimental results. As indicated by the effect of the change in the $\mathbf{F A} / \mathbf{1}$ ratio on the peak of $\mathbf{X}$, the change in surface properties could be due to decomposition of FA on the catalyst surface and active $\mathrm{H}$ adsorption on $\mathrm{Pd}$ or the $\mathrm{Al}_{2} \mathrm{O}_{3}$ support. Changes in the early time of transfer hydrogenation of nitroarenes have already been reported in the literature. Ebitani and co-workers ${ }^{9 c}$ attributed the color change of the catalyst within a few minutes of reaction to the in situ reduction of $\mathrm{Pd}(\mathrm{II})$ to $\mathrm{Pd}(0)$ by formic acid, while Ballauf's group ${ }^{5 \mathrm{e}-\mathrm{g}}$ related the short initial 
delay in which no reaction takes place to a rearrangement of the surface atoms. These induction periods were not accompanied by a change in selectivity. However, in a study involving 2-propanol as the hydrogen donor and an iridiumbased homogeneous catalyst, Chen and co-workers observed that the direct route and condensation route coexist for a while, and then the azo derivative slowly converts into aniline over a long period of time. ${ }^{22}$

\section{ATTEMPTS TO IDENTIFY THE INTERMEDIATE PRODUCT X}

Various attempts were made to identify the nature of $\mathbf{X}$. At first, since the UV spectrum of $\mathbf{X}$ has a huge absorption band around $250 \mathrm{~nm}$, intermediates involved in the mechanism of hydrogen transfer of nitroarenes that exhibit such a band were sought. The intermediates of the direct route are $p$-nitrosophenol (3) and p-hydroxylaminophenol (4). 3 is a commercial product and has a strong absorption band at 300 $\mathrm{nm}$. The condensation route involves $4,4^{\prime}$-azoxydiphenol (5), 4,4'-azodiphenol (6), and 4,4'-hydrazobisphenol (7) (Figure $1)$. We succeeded in synthesizing 6 by modifying a method described in the literature, ${ }^{11}$ and its maximum absorption band is around $360 \mathrm{~nm}$. In addition, according to Lerner, ${ }^{21} p$ benzoquinone imine (9) or its adducts identified as purple dyes could be formed in the reaction mixture, but 9 is not stable enough to be isolated. ${ }^{21}$ We also failed to synthesize the other intermediates 4,5 , and 7 . The formylated product, $N$-(4hydroxyphenyl)formamide (12), was also synthesized for comparison and has a medium absorption band at $246 \mathrm{~nm} .{ }^{25}$ Thus, by comparison of the UV spectra, $\mathbf{X}$ is none of the prepared compounds 3, 4, 6, or 12 .

In further experiments, NMR spectra and mass spectrometry (MS, ES+) patterns of mixtures rich in $\mathbf{X}$ were analyzed (Figures S8-S14). For this purpose, samples taken at the outlet of the reactor were collected at regular time intervals over $2 \mathrm{~min}$, while the composition of the reactor effluent was monitored with the on-line UV PDA. As can be seen in Figure 5 , the curve of $\mathbf{X}$ reaches its maximum in the sample taken at $25 \mathrm{~min}$ after the beginning of the reaction sequence.

First of all, we look for peaks whose UV intensity is the highest in the sample taken at $25 \mathrm{~min}$, in which the

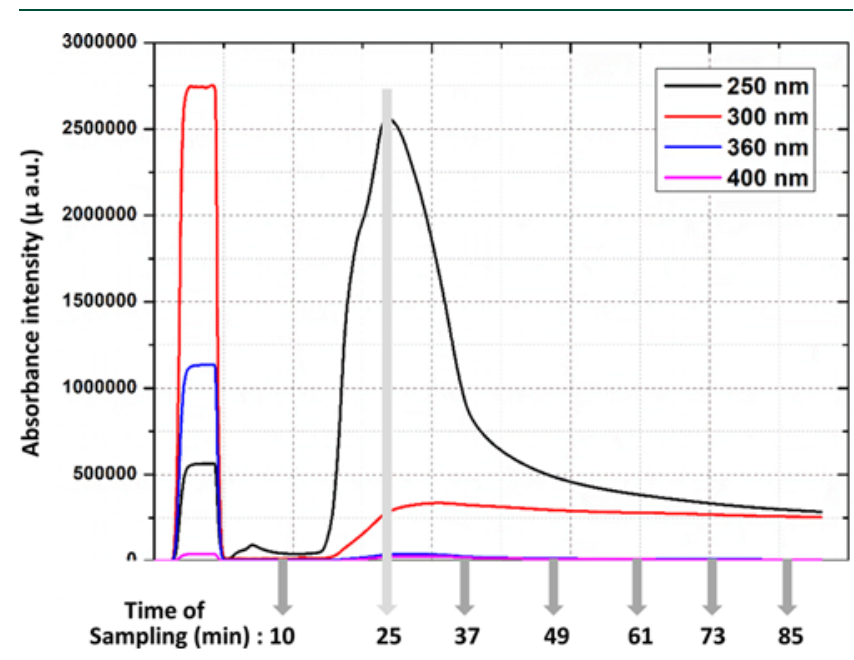

Figure 5. UV profiles of samples collected at different reaction times and then analyzed by MS and ${ }^{1} \mathrm{H}$ NMR spectroscopy. Conditions: $C_{1}^{0}$ $=0.5 \mathrm{mmol} / \mathrm{L} ; C_{\mathrm{FA}}^{0}=3 \mathrm{mmol} / \mathrm{L} ; Q=0.5 \mathrm{~mL} / \mathrm{min} ; T=25{ }^{\circ} \mathrm{C}$. concentration of $\mathbf{X}$ should be the maximum. Then we look for peaks whose intensity varies significantly from the feed, which are shown in Figure 6. As expected, the intensity of $\mathrm{m} / \mathrm{z}$

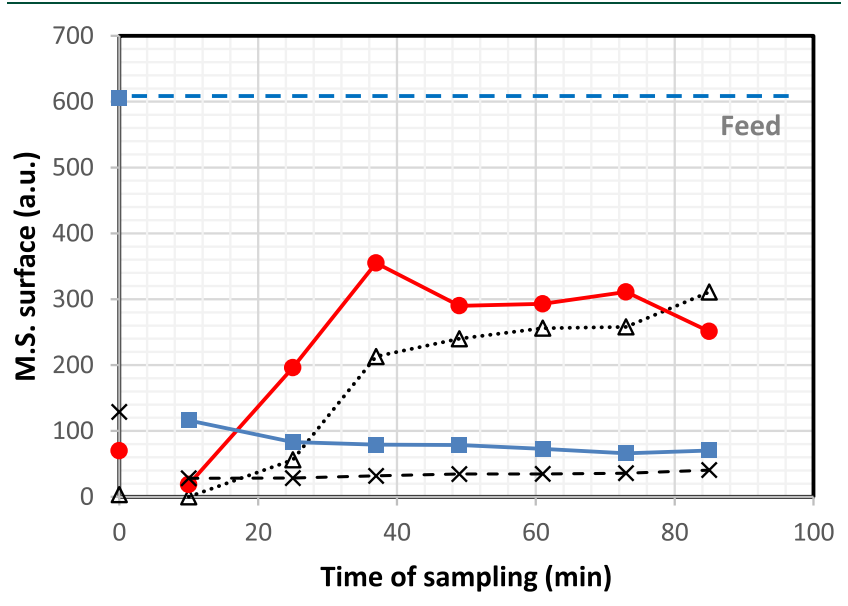

Figure 6. Intensity of the MS peaks of samples taken in the feed mixture (points at $t=0$ ) and at the outlet of the reactor at different times (ES+ mode): $\mathbf{\square}, m / z 140$ (1);,$m / z 110$ (2); $\times, m / z 124 ; \triangle$, $m / z 108$.

140 fragment peaks, corresponding to 1 , are lower at the exit of the reactor than in the reactor feed. One other peak, with $\mathrm{m} / \mathrm{z}$ 124 , exhibits the same trend. It could correspond to ionization of 3 (molar mass $=123 \mathrm{~g} / \mathrm{mol}$ ) and is also present in the feed analysis. Thus, it is possibly produced both during MS analysis and as an intermediate of the reaction. Only two $m / z$ values are significantly higher after reaction than in the feed: $m / z 110$, corresponding to the ionization of 2 , as expected, and $m / z 108$, which could be ionized $9(107 \mathrm{~g} / \mathrm{mol}) .2$ increases after a delay, as observed by HPLC. According to Lerner ${ }^{21}$ and Parida, ${ }^{18} \mathbf{9}$ could be formed from $\mathbf{2}$ or $\mathbf{4}$ in the reaction mixture and could also come from decomposition of $\mathbf{X}$ during the time elapsed between sampling and MS analysis. However, the sample collected at $t=25 \mathrm{~min}$, which should contain the highest concentration of $\mathbf{X}$, does not exhibit any intense peak.

A similar experiment was carried out with $\mathrm{D}_{2} \mathrm{O}$ as the solvent so that the samples could be analyzed by ${ }^{1} \mathrm{H}$ NMR spectroscopy. As shown in Figure 7, in the sample containing the highest $\mathbf{X}$ concentration, 1, 2, and FA are well-identified. There are several additional small peaks with $\delta$ between 6.89 and 7.22 that could correspond to $\mathbf{X}$. The reproducibility of these peaks was verified by repeating the same experiment. They do not match with the ${ }^{1} \mathrm{H}$ NMR spectra of 3 or 6 , but they could correspond to $\mathbf{1 2}$.

As we failed to undoubtedly analyze $\mathbf{X}$ by NMR spectroscopy or MS, we tried to observe it with on-line HPLC analysis. Figure 8 shows the series of chromatograms measured directly at the exit of the reactor during a reaction sequence. At the time corresponding to the maximum $\mathbf{X}$ concentration, a small HPLC peak (red, RT $=3.10 \mathrm{~min}$ ), attributed to the intermediate $\mathbf{X}$, appears between the peaks for $\mathbf{1}$ and $\mathbf{2}$ and then disappears in the following chromatograms.

This peak was not detected with off-line HPLC, which involves a step of collection of samples, i.e., a delay between reaction and analysis. With NMR spectroscopy as well as with MS, the signal that could be attributed to $\mathbf{X}$ is very weak and therefore difficult to analyze with accuracy. This could indicate that the intermediate $\mathbf{X}$ is quite unstable. Thus, the stability of 


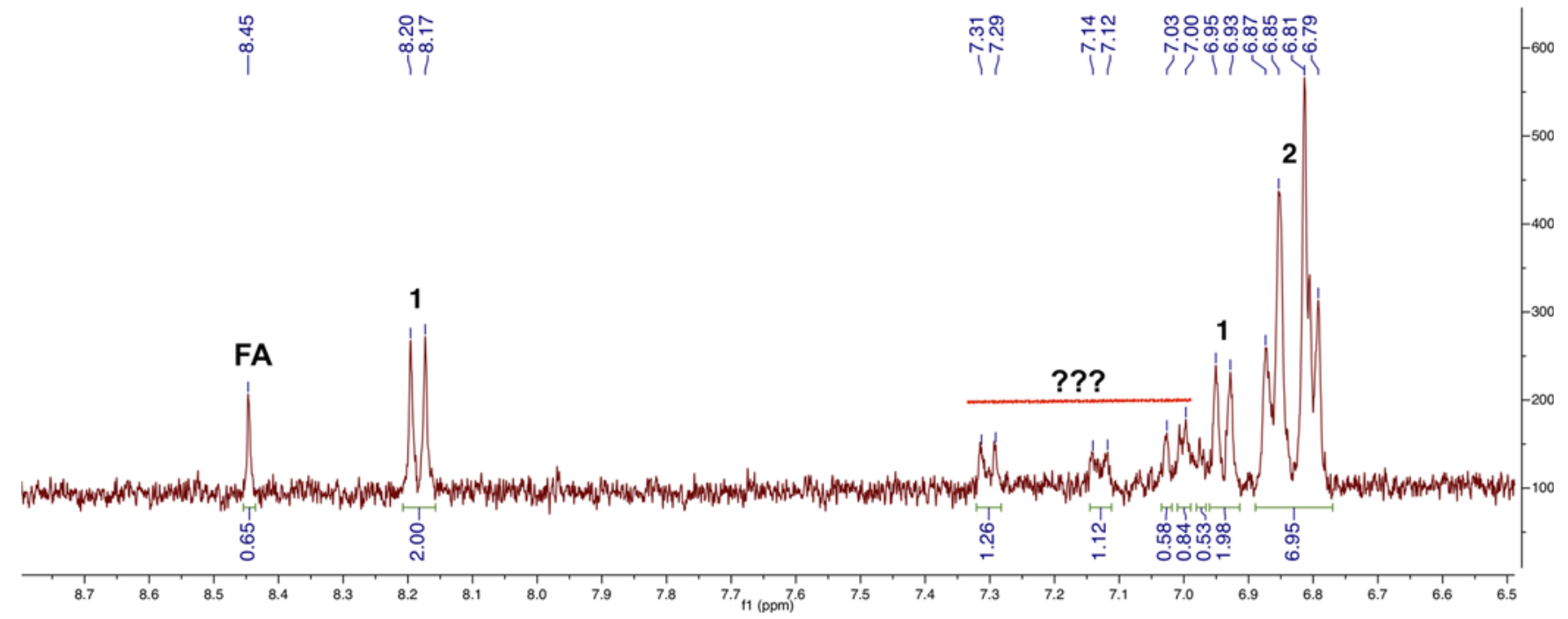

Figure 7. ${ }^{1} \mathrm{H}$ NMR spectrum of the sample containing $\mathrm{X}$. Conditions: $C_{1}^{0}=0.5 \mathrm{mmol} / \mathrm{L} ; C_{\mathrm{FA}}^{0}=3 \mathrm{mmol} / \mathrm{L} ; Q=0.5 \mathrm{~mL} / \mathrm{min} ; T=25^{\circ} \mathrm{C}$.

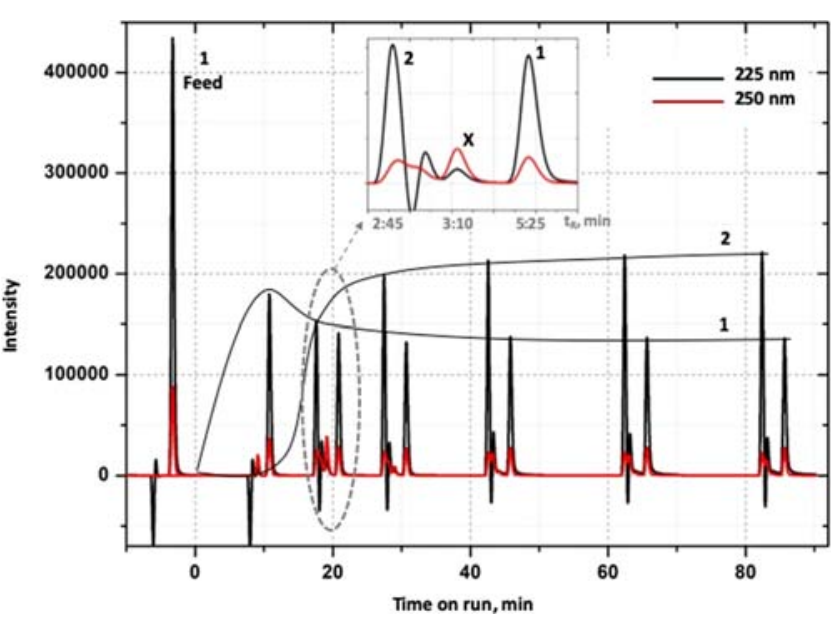

Figure 8. Investigation with on-line HPLC analysis of the reactor effluent. Conditions: $C_{1}^{0}=0.6 \mathrm{mmol} / \mathrm{L} ; C_{\mathrm{FA}}^{0}=1.8 \mathrm{mmol} / \mathrm{L} ; Q=1$ $\mathrm{mL} / \mathrm{min} ; \mathrm{T}=40{ }^{\circ} \mathrm{C}$.

$\mathbf{X}$ was evaluated in the following experiment: A normal flow reaction sequence was started, and the reactor effluent was continuously monitored with on-line UV spectroscopy. The pump was stopped when $\mathbf{X}$ reached its maximum. Thus, the spectra of the reaction mixture remaining in the UV cell were recorded until the stabilization. Figure 9 shows that intensity at $250 \mathrm{~nm}$ decreases rapidly, which clearly demonstrates the instability of $\mathbf{X}$, with a half-life of about $3 \mathrm{~min}$. This is the reason why only traces of $\mathbf{X}$ are detected by methods involving a delay between reaction and analysis.

In addition, it was observed that the mixture composition at the end of this experiment was composed of $\mathbf{2}$ and unreacted $\mathbf{1}$ only, in quite the same amounts as in the mixture obtained at the steady state of the flow reaction. This confirms that $\mathbf{X}$ may react with FA out of the reactor to be transformed in 2 (see $p$ S19 in the Supporting Information). Control experiments with the reaction of 2 alone or in the presence of FA ( 3 equiv) in the presence of the catalyst demonstrated its stability.

Additional UV-spectra calculations of possible intermediates 4-9, 10', and 11 were realized (Figure 10 and pp S20-S22 in the Supporting Information). Among them, the simulation of $8^{\prime}$ and 9 exhibits a strong absorption band around $250 \mathrm{~nm}$.

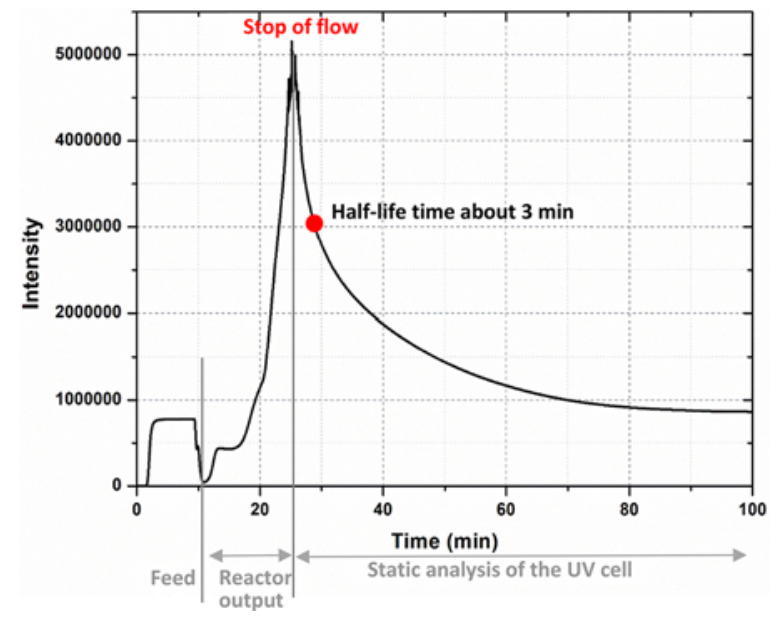

Figure 9. Evaluation of the stability of $\mathbf{X}$ in the UV cell. The intensity at $250 \mathrm{~nm}$ is shown as a function of time. Conditions: $C_{1}^{0}=0.6 \mathrm{mmol} /$ $\mathrm{L} ; C_{\mathrm{FA}}^{0}=1.8 \mathrm{mmol} / \mathrm{L} ; T=40^{\circ} \mathrm{C} ; Q=1 \mathrm{~mL} / \mathrm{min}$.<smiles>CN(O)c1ccc(O)cc1</smiles>

8 '<smiles>N=C1C=CC(=O)C=C1</smiles>

9<smiles>Oc1ccc(O)cc1</smiles><smiles>O=Nc1ccc(O)cc1</smiles>

Figure 10. Possible intermediates for the reduction of $\mathbf{1}$.

Moreover, components of the same molecular weight were detected by mass spectrometry. Therefore, as mentioned in previous studies, ${ }^{5 \mathrm{i}, 15,17,18}$ they could correspond to unstable $\mathbf{X}$.

To sum up, it was observed that the reaction may proceed according to at least two competitive reaction paths (Figure 11 ), one of them involving a transient unstable component that is observable by on-line UV, which we have called $\mathbf{X}$. The route via $\mathbf{X}$ formation is favored at the beginning of the reaction sequence and promoted by the deficit of FA. As soon as the catalyst equilibrates with the reaction mixture, the $\mathbf{X}$ route becomes less favored, leading to the release of $\mathbf{X}$ out of the reactor. Comparison of different reaction schemes by 


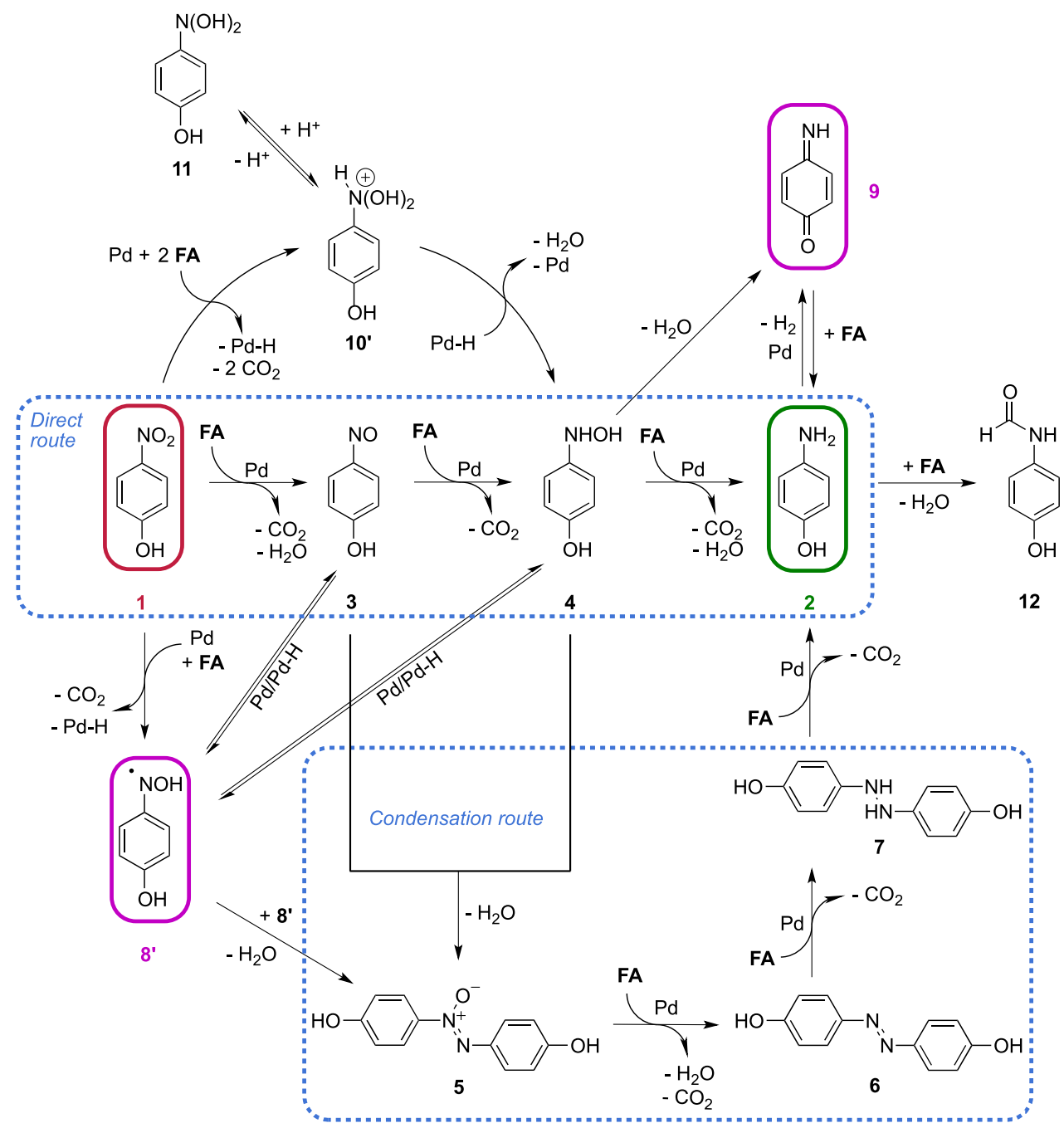

Figure 11. Overview of the possible paths allowing the formation of $\mathbf{2}$ with the presence of the transient observed molecules $\mathbf{8}^{\prime}$ and $\mathbf{9}$.

numerical simulation indicated that the change in the prevalence of the routes must be related to a change in catalyst reactivity. This change is most probably due to the production of active hydrogen on the catalyst. The structure of $\mathbf{X}$ was not strictly elucidated. It was only shown that it is not the nitroso, azo, or formamide derivative. The strong UV absorbance suggests several $\pi$-conjugated electrons, which could correspond to $\mathbf{8}^{\prime}$ or $\mathbf{9}$. It was also observed that $\mathbf{X}$ transforms to 2 without catalyst.

9 closely fits the observations. According to the literature, 2 oxidizes easily to $\mathbf{9}$, and this reaction is reversible in a redox medium. ${ }^{5 a, 17,18,26}$ Thus, at the beginning of the reaction sequence, the first molecules of $\mathbf{2}$ formed could transform to $\mathbf{9}$, giving two hydrogen atoms, until the active hydrogen concentration on the catalyst is in equilibrium with the FA concentration. After that, the reduction of 9 to 2 becomes dominant, and 9 is no longer formed. It can be noted that in this reaction scheme, $\mathbf{X}$ results in a successive reversible reaction and is not involved in the classical mechanism of hydrogenation of nitroarene. $\mathbf{8}^{\prime}$ could also correspond to $\mathbf{X}$. It has been postulated ${ }^{16}$ that it can react with itself to produce $\mathbf{5}$ with elimination of water without the assistance of any catalyst. However, $\mathbf{5}$ has not been observed outside of the reactor.

\section{CONCLUSION}

The experimental setup combining a catalytic fixed-bed microreactor, a photodiode array detector, and an HPLC device has been shown to be particularly useful in the study of complex reactions such as transfer hydrogenation of 1 with FA. With on-line HPLC analysis, the quantitative analysis of both $\mathbf{1}$ and 2 can be realized, and therefore, the time-concentration profile of these components can be obtained in aqueous acidic media, which has not been done previously. The simultaneous quantification of $\mathbf{1}$ and $\mathbf{2}$ indicates that within the experimental error range, $\mathbf{2}$ is the only product of the reaction at steady state. On-line UV analysis highlights the observable transient production of a quite unstable intermediate species, $\mathbf{X}$, characterized by a strong absorbance at $250 \mathrm{~nm}$. These experimental observations, associated with comparison of two reaction schemes by numerical simulation, allowed the $\mathbf{X}$ transient formation to be related to a change in the active hydrogen coverage of the catalyst. Additional UV-spectra calculations support the proposition of a new mechanism in the transfer hydrogenation of $\mathbf{1}$ to $\mathbf{2}$ involving $\mathbf{8}^{\prime}$ or $\mathbf{9}$ not as a reaction intermediate but as a successive byproduct.

The usefulness of the experimental setup should be emphasized because only the dynamic study of the reaction under transient conditions, coupled with $3 \mathrm{D}$ on-line UV analysis, allowed the clear observation of the formation of the 
transient component $\mathbf{X}$ and its possible identification as $\mathbf{8}^{\prime}$ or 9. Knowing the complexity of this reaction, further kinetic studies will be realized on various oxide supports in order to compare their effects on the reaction pathways.

(1) Mason, B. P.; Price, K. E.; Steinbacher, J. L.; Bogdan, A. R.; McQuade, D. T. Greener Approaches to Organic Synthesis Using Microreactor Technology. Chem. Rev. 2007, 107 (6), 2300-2318.

(2) Tanimu, A.; Jaenicke, S.; Alhooshani, K. Heterogeneous Catalysis in Continuous Flow Microreactors: A Review of Methods and Applications. Chem. Eng. J. 2017, 327, 792-821.

(3) Formenti, D.; Ferretti, F.; Scharnagl, F. K.; Beller, M. Reduction of Nitro Compounds Using 3d-Non-Noble Metal Catalysts. Chem. Rev. 2019, 119 (4), 2611-2680.

(4) Blaser, H.-U. A Golden Boost to an Old Reaction. Science 2006, 313, 312-313.

(5) Reviews: (a) Zhao, P.; Feng, X.; Huang, D.; Yang, G.; Astruc, D. Basic Concepts and Recent Advances in Nitrophenol Reduction by Gold- and Other Transition Metal Nanoparticles. Coord. Chem. Rev. 2015, 287, 114-136. Selected examples: (b) Saha, S.; Pal, A.; Kundu, S.; Basu, S.; Pal, T. Photochemical Green Synthesis of CalciumAlginate-Stabilized $\mathrm{Ag}$ and $\mathrm{Au}$ Nanoparticles and Their Catalytic Application to 4-Nitrophenol Reduction. Langmuir 2010, 26 (4), 2885-2893. (c) Katiyar, S.; Mondal, K.; Sharma, A. One-Step SolGel Synthesis of Hierarchically Porous, Flow-through Carbon/Silica Monoliths. RSC Adv. 2016, 6 (15), 12298-12310. (d) Jiang, Z.; Xie, J.; Jiang, D.; Jing, J.; Qin, H. Facile Route Fabrication of Nano-Ni Core Mesoporous-Silica Shell Particles with High Catalytic Activity towards 4-Nitrophenol Reduction. CrystEngComm 2012, 14 (14), 4601-4611. (e) Gu, S.; Wunder, S.; Lu, Y.; Ballauff, M.; Fenger, R.;
Rademann, K.; Jaquet, B.; Zaccone, A. Kinetic Analysis of the Catalytic Reduction of 4-Nitrophenol by Metallic Nanoparticles. J. Phys. Chem. C 2014, 118 (32), 18618-18625. (f) Gu, S.; Kaiser, J.; Marzun, G.; Ott, A.; Lu, Y.; Ballauff, M.; Zaccone, A.; Barcikowski, S.; Wagener, P. Ligand-Free Gold Nanoparticles as a Reference Material for Kinetic Modelling of Catalytic Reduction of 4-Nitrophenol. Catal. Lett. 2015, 145 (5), 1105-1112. (g) Gu, S.; Lu, Y.; Kaiser, J.; Albrecht, M.; Ballauff, M. Kinetic Analysis of the Reduction of 4Nitrophenol Catalyzed by $\mathrm{Au} / \mathrm{Pd}$ Nanoalloys Immobilized in Spherical Polyelectrolyte Brushes. Phys. Chem. Chem. Phys. 2015, 17 (42), 28137-28143. (h) Feng, J.; Wang, Q.; Fan, D.; Ma, L.; Jiang, D.; Xie, J.; Zhu, J. Nickel-Based Xerogel Catalysts: Synthesis via Fast Sol-Gel Method and Application in Catalytic Hydrogenation of $p$ Nitrophenol to $p$-Aminophenol. Appl. Surf. Sci. 2016, 382, 135-143. (i) Thambiliyagodage, C. J.; Hakat, Y.; Bakker, M. G. One Pot Synthesis of Carbon/Ni Nanoparticle Monolithic Composites by Nanocasting and Their Catalytic Activity for 4-Nitrophenol Reduction. Curr. Catal. 2016, 5 (2), 135-146. (j) Bae, S.; Gim, S.; Kim, H.; Hanna, K. Effect of NaBH4 on Properties of Nanoscale Zero-Valent Iron and Its Catalytic Activity for Reduction of pNitrophenol. Appl. Catal., B 2016, 182, 541-549. (k) Liu, A.; Yang, L.; Traulsen, C. H.-H.; Cornelissen, J. J. L. M. Immobilization of Catalytic Virus-like Particles in a Flow Reactor. Chem. Commun. 2017, 53 (54), 7632-7634. (1) Uberman, P. M.; García, C. S.; Rodríguez, J. R.; Martín, S. E. PVP-Pd Nanoparticles as Efficient Catalyst for Nitroarene Reduction under Mild Conditions in Aqueous Media. Green Chem. 2017, 19 (3), 739-748. (m) Jiang, S.-F.; Ling, L.-L.; Xu, Z.; Liu, W.-J.; Jiang, H. Enhancing the Catalytic Activity and Stability of Noble Metal Nanoparticles by the Strong Interaction of Magnetic Biochar Support. Ind. Eng. Chem. Res. 2018, 57 (39), 13055-13064.

(6) Javaid, R.; Kawasaki, S.; Suzuki, A.; Suzuki, T. M. Simple and Rapid Hydrogenation of $p$-Nitrophenol with Aqueous Formic Acid in Catalytic Flow Reactors. Beilstein J. Org. Chem. 2013, 9 (129), 11561163.

(7) Corma, A.; Serna, P. Chemoselective Hydrogenation of Nitro Compounds with Supported Gold Catalysts. Science 2006, 313 (5785), 332-334.

(8) Joncour, R.; Ferreira, A.; Duguet, N.; Lemaire, M. Preparation of Para -Aminophenol from Nitrobenzene through Bamberger Rearrangement Using a Mixture of Heterogeneous and Homogeneous Acid Catalysts. Org. Process Res. Dev. 2018, 22 (3), 312-320.

(9) (a) Czulak, J.; Jouannin, C.; Vincent, T.; Dez, I.; Gaumont, A.C.; Guibal, E. Nitrophenol Hydrogenation Using Pd Immobilized on Ionic Liquid-Alginate Spherical Resins. Sep. Sci. Technol. 2012, 47 (14-15), 2166-2176. (b) Verho, O.; Nagendiran, A.; Tai, C.-W.; Johnston, E. V.; Bäckvall, J.-E. Nanopalladium on Amino-Functionalized Mesocellular Foam as an Efficient and Recyclable Catalyst for the Selective Transfer Hydrogenation of Nitroarenes to Anilines. ChemCatChem 2014, 6 (1), 205-211. (c) Tuteja, J.; Nishimura, S.; Ebitani, K. Base-Free Chemoselective Transfer Hydrogenation of Nitroarenes to Anilines with Formic Acid as Hydrogen Source by a Reusable Heterogeneous Pd/ZrP Catalyst. RSC Adv. 2014, 4 (72), 38241-38249. (d) Chaiseeda, K.; Nishimura, S.; Ebitani, K. Gold Nanoparticles Supported on Alumina as a Catalyst for Surface Plasmon-Enhanced Selective Reductions of Nitrobenzene. ACS Omega 2017, 2 (10), 7066-7070. (e) Li, X.-H.; Cai, Y.-Y.; Gong, L.-H.; Fu, W.; Wang, K.-X.; Bao, H.-L.; Wei, X.; Chen, J.-S. Photochemically Engineering the Metal-Semiconductor Interface for Room-Temperature Transfer Hydrogenation of Nitroarenes with Formic Acid. Chem. - Eur. J. 2014, 20, 16732-16737.

(10) Luo, Q.; Wang, T.; Beller, M.; Jiao, H. Hydrogen Generation from Formic Acid Decomposition on $\mathrm{Ni}(211), \mathrm{Pd}(211)$ and $\mathrm{Pt}(211)$. J. Mol. Catal. A: Chem. 2013, 379, 169-177.

(11) Li, X.-H.; Cai, Y.-Y.; Gong, L.-H.; Fu, W.; Wang, K.-X.; Bao, H.L.; Wei, X.; Chen, J.-S. Photochemically Engineering the MetalSemiconductor Interface for Room-Temperature Transfer Hydrogenation of Nitroarenes with Formic Acid. Chem. - Eur. J. 2014, 20 (50), 16732-16737. 
(12) Song, J.; Huang, Z.-F.; Pan, L.; Li, K.; Zhang, X.; Wang, L.; Zou, J.-J. Review on Selective Hydrogenation of Nitroarene by Catalytic, Photocatalytic and Electrocatalytic Reactions. Appl. Catal., B 2018, 227, 386-408.

(13) Corma, A.; Concepción, P.; Serna, P. A Different Reaction Pathway for the Reduction of Aromatic Nitro Compounds on Gold Catalysts. Angew. Chem., Int. Ed. 2007, 46 (38), 7266-7269.

(14) Figueras, F.; Coq, B. Hydrogenation and Hydrogenolysis of Nitro-, Nitroso-, Azo-, Azoxy- and Other Nitrogen-Containing Compounds on Palladium. J. Mol. Catal. A: Chem. 2001, 173 (12), 223-230.

(15) Gelder, E. A.; Jackson, S. D.; Lok, C. M. The Hydrogenation of Nitrobenzene to Aniline: A New Mechanism. Chem. Commun. 2005, $4,522-524$.

(16) Zhang, L.; Shao, Z.-J.; Cao, X.-M.; Hu, P. Insights into Different Products of Nitrosobenzene and Nitrobenzene Hydrogenation on $\operatorname{Pd}(111)$ under Realistic Reaction Conditions. J. Phys. Chem. C 2018, 122 (35), 20337-20350.

(17) Abd El Maksod, I. H.; Saleh, T. S. The Use of Nano Supported Nickel Catalyst in Reduction of $p$-Nitrophenol Using Hydrazine as Hydrogen Donor. Green Chem. Lett. Rev. 2010, 3 (2), 127-134.

(18) Rana, S.; Parida, K. M. A Simple and Efficient Protocol Using Palladium Based Lacunary Phosphotungstate Supported Mesoporous Silica towards Hydrogenation of P-Nitrophenol to p-Aminophenol at Room Temperature. Catal. Sci. Technol. 2012, 2 (5), 979-986.

(19) Honeychurch, K. C.; Hart, J. P. Voltammetric Behavior OfpNitrophenol and Its Trace Determination in Human Urine by Liquid Chromatography with a Dual Reductive Mode Electrochemical Detection System. Electroanalysis 2007, 19 (21), 2176-2184.

(20) Conant, J. B.; Pratt, M. F. The Irreversible Oxidation Of Organic Compounds I. The Oxidation Of Aminophenols By Reagents Of Definite Potential. J. Am. Chem. Soc. 1926, 48 (12), 3178-3192.

(21) Lerner, L. Identity of a Purple Dye Formed by Peroxidic Oxidation of $p$-Aminophenol at Low pH. J. Phys. Chem. A 2011, 115 (35), 9901-9910.

(22) Chen, S.; Lu, G.; Cai, C. Iridium-Catalyzed Transfer Hydrogenation of Nitroarenes to Anilines. New J. Chem. 2015, 39 (7), 5360-5365.

(23) Serna, P.; Corma, A. Transforming Nano Metal Nonselective Particulates into Chemoselective Catalysts for Hydrogenation of Substituted Nitrobenzenes. ACS Catal. 2015, 5 (12), 7114-7121.

(24) (a) Karimi, B.; Mansouri, F.; Vali, H. A Highly WaterDispersible/Magnetically Separable Palladium Catalyst: Selective Transfer Hydrogenation or Direct Reductive N-Formylation of Nitroarenes in Water. ChemPlusChem 2015, 80 (12), 1750-1759. (b) Nasir Baig, R. B.; Verma, S.; Nadagouda, M. N.; Varma, R. S. A Photoactive Bimetallic Framework for Direct Aminoformylation of Nitroarenes. Green Chem. 2016, 18 (4), 1019-1022. (c) Dong, X.; Wang, Z.; Duan, Y.; Yang, Y. One-Pot Selective N -Formylation of Nitroarenes to Formamides Catalyzed by Core-Shell Structured Cobalt Nanoparticles. Chem. Commun. 2018, 54 (64), 8913-8916. (d) Wang, Y.; Zhan, Z.; Zhou, Y.; Lei, M.; Hu, L. A Green, Efficient, and Rapid Procedure for the Hydrogenation of Nitroarenes to Formanilides in Water. Monatsh. Chem. 2018, 149 (3), 527-533.

(25) Hosseini-Sarvari, M.; Sharghi, $\mathrm{H}$. $\mathrm{ZnO}$ as a New Catalyst for $\mathrm{N}$ Formylation of Amines under Solvent-Free Conditions. J. Org. Chem. 2006, 71 (17), 6652-6654.

(26) (a) Rau, N. J.; Welles, E. A.; Wenthold, P. G. Anionic Substituent Control of the Electronic Structure of Aromatic Nitrenes. J. Am. Chem. Soc. 2013, 135 (2), 683-690 and references therein. (b) Mokhtari, B.; Nematollahi, D.; Salehzadeh, H. Paired Electrochemical Conversion of Nitroarenes to Sulfonamides, Diarylsulfones and Bis(Arylsulfonyl)Aminophenols. Green Chem. 2018, 20 (7), 1499-1505. 